\title{
Photopsias are related in part to perceived stress and positive mood in retinitis pigmentosa
}

AK Bittner ${ }^{1}$, JA Haythornthwaite ${ }^{2}$, M Diener-West ${ }^{3}$ and G Dagnelie ${ }^{1}$

\begin{abstract}
Purpose We measured the relationship between the occurrence of photopsias (spontaneous phosphenes), and retinitis pigmentosa (RP) subjects' level of vision, light exposure, and psychosocial factors to attempt to confirm RP patients' previous reports of

Conclusions Increased photopsias appear to be related to times when subjects report increased perceived stress and/or decreased positive mood, rather than RP patients' age, level of vision, reported light exposure, or sleepiness.

Eye (2012) 26, 101-108; doi:10.1038/eye.2011.247; published online 14 October 2011
\end{abstract} these associations.

Methods A total of 36 RP subjects self-administered PC-based binocular visual acuity, contrast sensitivity, and visual field tests at home twice a week, for 16 sessions in 2-3 months. After each session, subjects reported photopsias during the vision tests and completed questionnaires: Epworth Sleepiness Scale, Stanford Sleepiness Scale, Perceived Stress Scale, and Positive and Negative Affect Schedules.

Results Across all subjects, photopsias occurred during $47 \%$ of sessions. Five (14\%) subjects never noted photopsias, while five others noted photopsias at every session. Two-thirds of subjects experienced photopsias frequently ( $>20 \%$ of sessions). On average, the odds of noticing photopsias increased by $57 \%$ for every 1-point increase in mean perceived stress $(\mathrm{OR}=1.57 ; 95 \% \mathrm{CI}$ : 1.04 , $2.4 ; P=0.03)$ or reduced by $38 \%$ for every 1 -point increase in positive $\operatorname{mood}(O R=0.62$; 95\% CI: $0.39,0.98 ; P=0.04)$, after adjusting for age, gender, and vision. Similarly, the odds of experiencing photopsias during a session increased by $16 \%$ for every 3-point increase in perceived stress and decreased by $17 \%$ for every 3-point increase in positive mood, after adjusting for age and gender $(\mathrm{OR}=1.16$; 95\% CI: 1.01, 1.33; $P=0.048)(\mathrm{OR}=0.83 ; 95 \%$ CI: $0.73,0.94 ; P=0.004)$, respectively. Frequency of photopsias was not statistically significantly related to other factors measured.
Keywords: photopsias; phosphenes; flashes; stress; mood; retinitis pigmentosa

Introduction

Simple visual pseudohallucinations are also known as phosphenes or photopsias, and consist of unformed, geometric patterns or light phenomena. In photoreceptor disease, these phenomena have been described as many widespread flickering, pulsating, or shimmering lights (snow on a TV screen, as opposed to single flashes). ${ }^{1}$ Retinitis pigmentosa (RP) patients have previously confirmed their experiences of these phenomena in survey research, ${ }^{2,3}$ most commonly described as slow localized dots or shapes involving semicircles, but also as quick flashes of light, static noise, or fluorescence. In a previous survey of patients with retinal vision loss, the majority reported spontaneous visual phenomena, with simple phenomena occurring more frequently than complex phenomena involving formed images. ${ }^{4}$ In the present study, we focused on the more common, simple visual pseudohallucinations or photopsias in RP.

Photopsias tend to increase in the absence of light and visual stimuli, and therefore it would seem logical to hypothesize that RP patients with more advanced vision loss would experience photopsias more frequently. However, there is at least one reference acknowledging that visual delusions may be
${ }^{1}$ Johns Hopkins University; Wilmer Eye Institute, Baltimore, MD, USA

${ }^{2}$ Department of Psychiatry and Behavioral Sciences, Johns Hopkins University, Baltimore, MD, USA

${ }^{3}$ Department of Biostatistics, Johns Hopkins Bloomberg School of Public Health, Baltimore, MD, USA

Correspondence: AK Bittner Johns Hopkins University, Wilmer Eye Institute, $550 \mathrm{~N}$ Broadway, 6th floor, Baltimore, MD 21205, USA Tel: + 1410502 6430; Fax + 14109551829 . E-mail: abittne1@jhmi.edu

Received: 27 April 2011 Accepted in revised form: 9 August 2011

Published online: 14 October 2011

Presented at the 21st annual Wilmer Research Meeting in Baltimore, MD, USA on 23 April 2010, and the Envision Conference in San Antonio, TX, USA on 25 September 2010. 
a subtle precursor of impending vision loss, ${ }^{5}$ and about half of RP patients surveyed reported having photopsias at early stages, even before they were diagnosed with $\mathrm{RP}^{3}$ Therefore, we were interested in relating RP patients' level of measured vision to their frequency of photopsias.

In a previous survey, RP patients indicated that the top three factors that tend to increase photopsias were bright light, fatigue, and stress. ${ }^{3}$ However, we were unable to identify any previously published studies that attempted to systematically measure these relationships in RP patients. We therefore set out to determine: (1) whether RP subjects experienced increased photopsias during a series of self-administered vision tests at times when their perceived stress, negative mood, sleepiness, or light exposure was greater, or (2) whether subjects who had higher mean levels of stress, negative mood, or sleepiness were prone to note photopsias more frequently.

\section{Methods}

Institutional Review Board/Ethics Committee approval was obtained. Informed consent was obtained from the subjects after explanation of the nature and possible consequences of the study. This research is HIPAA compliant and adhered to the tenets of the Declaration of Helsinki. Data collection occurred from December 2008 through April 2010.

\section{Subjects}

Study participants included 36 individuals diagnosed with RP. The majority of the subjects $(n=24 ; 67 \%)$ were recruited through the clinical practices of low-vision optometrists and retinal specialists at the Johns Hopkins Wilmer Eye Institute. The remaining subjects selfreferred after learning of the study through online listings. Individuals were eligible for the study if they had a confirmed diagnosis of RP, were over age 18, and experienced any level of vision, provided they could read reverse contrast, large-sized font on the PC to complete the questionnaires and vision tests used in the study. Participants possessed basic computer skills at a minimum, and the study provided a loaner PC if they did not have regular access to one. Subjects were recruited with the premise that their ocular status was likely to remain relatively stable throughout a 2- to 3-month period, during which the testing occurred, and this was confirmed by the PC-based vision test results. The subjects did not have any significant cognitive deficits that would have interfered with their responses to the vision tests and questionnaires.

Subjects' self-reported duration of any type of uncorrectable vision loss other than night vision loss ranged from 1 to 51 years (mean 17; SD 13 years), while their self-reported duration of night vision loss ranged from 1 to 71 years (mean 27; SD 18 years). Exactly half of the subjects were female. All subjects' ages ranged from 20 to 77 , with a mean of 51 years. In all, $8 \%$ of the subjects were African-American, $11 \%$ were Hispanic, and $81 \%$ were Caucasian.

\section{Data collection}

A total of 33 of the 36 subjects enrolled at our center, and lab-based vision tests were performed at a single session. Best-corrected visual acuity (VA) was measured binocularly with the Early Treatment of Diabetic Retinopathy Study (ETDRS; Lighthouse International, New York, NY, USA) charts at $3 \mathrm{~m}$ or closer if fewer than 10 letters were identified. Best-corrected, binocular Pelli-Robson contrast sensitivity (CS) (Metropia Ltd, Harlow, UK) was assessed at $1 \mathrm{~m}$. Subjects had a mean ETDRS VA of 0.23 logMAR (SD 0.34, range -0.12, 0.98) and mean Pelli Robson CS of $1.3 \log$ CS (SD 0.6, range 0.05, 2.0). The visual field in each eye was measured using the Goldmann Visual Field (GVF) V4e and III4e test targets, and the eye with the larger isopter diameter was used for the analyses. Subjects had mean isopter diameters of $54^{\circ}$ (SD $52^{\circ}$, range $7^{\circ}, 149^{\circ}$ ) and $40^{\circ}\left(\mathrm{SD} 43^{\circ}\right.$, range $\left.5^{\circ}, 130^{\circ}\right)$ with the V4e and III4e test targets, respectively. All vision tests were performed by a single examiner (AKB). The remaining three subjects did not reside locally and were enrolled over the phone via oral consent, and their ocular health status and diagnosis of RP were verified by records forwarded from their local eye care provider.

Within 1-2 weeks of study enrollment, subjects were asked to initiate a series of PC-based vision tests and questionnaires that were self-administered at home. The PC-based vision tests included binocular VA, CS, and static VF. They were previously developed at our center and validated in RP subjects while on placebo during a clinical trial. ${ }^{6}$ The study protocol included 16 test sessions completed twice a week over a period of about 2-3 months. In all, 30 (83\%) of the subjects completed all of the 16 test sessions, while 3 completed 15, 1 completed 14, 1 completed 13, and 1 completed 10 sessions. To maximize the chances of capturing the full range of vision variability of vision during the day, the test times were randomized such that equal numbers of tests were taken in the morning before noon on weekends and weekdays (either Wednesday or Thursday), and in the evenings after 1800 hours on weekends and the same weekdays.

Immediately following the PC-based vision tests, the software administered a series of questionnaires. The first question inquired about photopsias or light show phenomena experienced during the vision test session. Subjects were asked if they noticed the following types of 
photopsias: white-out glare, slow phosphenes, quick flashes of light, static noise, and fluorescence or background glow. These descriptions of photopsias were previously identified during a survey of photopsias in $\mathrm{RP}^{3}$ More than one selection was permitted if participants noticed more than one type of photopsia.

Four standardized questionnaires to assess psychosocial factors were also administered after each PC test session after the question about photopsias: the Stanford Sleepiness Scale (SSS), ${ }^{7,8}$ Epworth Sleepiness Scale (ESS), ${ }^{9}$ Perceived Stress Scale (PSS), ${ }^{10}$ and the Positive and Negative Affect Scales (PANAS). ${ }^{11}$ The SSS consists of a single item 7-point scale to determine sleepiness at the time of test administration, and the ESS contains 8 items to inquire about subjects' tendency to doze during various activities on the day of the test.

For the PSS, subjects indicated the degree to which situations in their life are appraised as stressful, or how often they have found their lives unpredictable, uncontrollable, and overloaded in the last $24 \mathrm{~h}$. The questions are general in nature and hence relatively free of content that might be specific to any subpopulation group or topic. Levels of appraised stress measured by the PSS should be influenced by daily hassles, major events, and changes in coping resources. Also, it may include non-occurrence of events, expectations concerning future events, and stress from events occurring in the lives of friends and relatives. The PSS is not a diagnostic instrument, so there are no established cutoffs. The 5-point scale for each item in the PSS is: $0=$ never, $1=$ almost never, $2=$ sometimes, $3=$ fairly often, and $4=$ very often. Total scores for the 14 items can range from 0 to 56 . In a large probability sample of US adults who were asked about stress in the last month, the mean \pm SD for the PSS was 19.6 $\pm 7.5 .^{12}$

The PANAS provides a brief measure of mood, consisting of 10 items to assess positive mood (interested, excited, strong, enthusiastic, proud, alert, inspired, determined, attentive, and active) and 10 items to assess negative mood (distressed, upset, guilty, scared, hostile, irritable, ashamed, nervous, jittery, and afraid) in the past $24 \mathrm{~h}$, and each set is summed to create a score of positive or negative mood. The 5-point scale for each item in the PANAS is: $1=$ very slightly, $2=$ a little, $3=$ moderately, $4=$ quite a bit, and $5=$ extremely. Total scores for each set of 10 items can range from 10 to 50 . In a large sample of US adults (mean age 38 years, SD 13) who were asked at one occasion about mood states in the last few weeks, the mean \pm SD was $31.0 \pm 8.1$ for positive mood and 19.4 \pm 7.1 for negative mood. ${ }^{13}$ Another previous study in a large sample of college students reported that scores tended to be higher for both positive and negative mood on average by $\sim 3$ points when respondents rated mood over the past few weeks $v$ s today. ${ }^{11}$
Before each PC-based vision test, the participants indicated if they were exposed to bright, medium, or dim light in the previous hour. On a single occasion during the course of the study, participants completed the Beck Depression Inventory (BDI), ${ }^{14}$ which was mailed or emailed to them. The standard cutoffs for the BDI are: 0-9= minimal depression, $10-18=$ mild depression, 19-29= moderate depression, and 30-63= severe depression.

\section{Data analysis}

We defined subjects who experienced photopsias frequently during the PC-based vision tests as those with the presence of photopsias during $>20 \%$ of the test sessions. Two-sample $t$-tests were used to compare characteristics (mean age, psychosocial variables, and lab-based vision measures) of subjects who did and did not experience photopsias frequently. We determined the mean score across all test sessions for each psychosocial variable (perceived stress, positive and negative mood, and sleepiness), and used logistic regressions to determine odds ratios for experiencing photopsias frequently ( $>20 \%$; as a binary variable) in relation to each mean psychosocial variable and depression score.

Another aim of the study was to determine whether the presence or absence of photopsias as a binary variable during each session was related to each of the three psychosocial states assessed at the time of the session as a continuous score. These repeated measures data were fitted using multi-level, mixed effects models for logistic regression, with clustering by subject. Models assuming random intercepts as well as models assuming both random slopes and random intercepts were explored. The log likelihoods for these models were approximated by adaptive Gaussian quadrature. Data were analyzed using Stata/IC Version 10.0 (Stata Corp., College Station, TX, USA).

\section{Results}

Across all subjects, photopsias were indicated during $47 \%$ of test sessions. In all, 5 (14\%) subjects never noted photopsias, while $5(14 \%)$ subjects noted photopsias at every test session. A total of $24(67 \%)$ of the subjects experienced photopsias frequently ( $>20 \%$ of test sessions) during the PC-based vision tests. The most common type of photopsia was (a group/series of) quick flashes of light, noted during $17 \%$ of test sessions, followed by phosphenes, described as slow, localized dots or shapes, during $11 \%$ of sessions. White-out glare was noted at $8 \%$ of sessions, fluorescence or background glow was experienced at $6 \%$ of sessions, and static noise (like on a TV without reception) was seen at $5 \%$ of sessions. 
Table 1 RP subjects' characteristics: photopsias frequency across test sessions

\begin{tabular}{|c|c|c|c|c|}
\hline & $\begin{array}{c}\text { Frequency }<20 \% \\
(\mathrm{n}=12)\end{array}$ & $\begin{array}{c}\text { Frequency }>20 \% \\
(\mathrm{n}=24)\end{array}$ & $\begin{array}{c}\text { Total } \\
(\mathrm{n}=36)\end{array}$ & P-value \\
\hline \multicolumn{5}{|l|}{ Demographics } \\
\hline Gender-\% male & 16.7 & 66.7 & 50 & $0.005^{*}$ \\
\hline Age in years - mean $(\mathrm{SD})$ & $54.5(17.7)$ & $49.1(13.4)$ & $50.8(14.9)$ & 0.36 \\
\hline \multicolumn{5}{|l|}{ Psychosocial variables: mean (SD) } \\
\hline Perceived Stress Scale score (PSS) & $18.1(2.7)$ & $22.4(5.1)$ & $21.0(4.8)$ & $0.002^{*}$ \\
\hline Positive Mood score (PANAS) & $34.4(6.4)$ & $26.7(7.2)$ & $29.3(7.8)$ & $0.003^{*}$ \\
\hline Negative Mood score (PANAS) & $13.3(2.6)$ & $16.0(5.0)$ & $15.1(4.5)$ & $0.04^{*}$ \\
\hline Epworth Sleepiness Scale score (ESS) & $12.4(3.3)$ & $13.6(3.8)$ & $13.2(3.6)$ & 0.32 \\
\hline Stanford Sleepiness Scale score (SSS) & $2.2(0.8)$ & $2.5(0.7)$ & $2.4(0.8)$ & 0.34 \\
\hline Beck Depression Inventory score (BDI) & $5.8(4.2)$ & $11.6(9.6)$ & $10.0(8.8)$ & $0.03^{*}$ \\
\hline \multicolumn{5}{|l|}{ Vision: mean $(S D)$} \\
\hline ETDRS VA OU (logMAR) & $0.28(0.45)$ & $0.21(0.27)$ & $0.24(0.34)$ & 0.63 \\
\hline Pelli-Robson CS OU (log units) & $1.30(0.67)$ & $1.30(0.59)$ & $1.30(0.61)$ & 0.97 \\
\hline GVF diameter (V4e; degrees) & $47.1(50.3)$ & $48.1(48.1)$ & $47.8(48.1)$ & 0.96 \\
\hline
\end{tabular}

*Statistically significant $(P<0.05)$

Table 2 Crude and adjusted odds of increased frequency of photopsias (during $>20 \%$ of sessions) for each psychosocial variable

\begin{tabular}{|c|c|c|c|c|c|c|}
\hline \multirow[t]{2}{*}{ Psychosocial variable } & \multicolumn{3}{|c|}{ Crude } & \multicolumn{3}{|c|}{ Adjusted $^{\mathrm{a}}$} \\
\hline & OR & $(95 \% \mathrm{CI})$ & P-value & OR & $(95 \%$ CI $)$ & P-value \\
\hline Mean Perceived Stress Scale score (PSS) & 1.33 & $(1.05-1.7)$ & $0.02^{\mathrm{b}}$ & 1.57 & $(1.04-2.4)$ & $0.03^{\mathrm{b}}$ \\
\hline Mean Positive Mood score (PANAS) & 0.83 & $(0.72-0.96)$ & $0.01^{\mathrm{b}}$ & 0.62 & $(0.39-0.98)$ & $0.04^{\mathrm{b}}$ \\
\hline Mean Negative Mood score (PANAS) & 1.26 & $(0.97-1.6)$ & 0.09 & 1.44 & $(0.95-2.2)$ & 0.09 \\
\hline Mean Epworth Sleepiness Scale (ESS) & 1.11 & $(0.90-1.4)$ & 0.33 & 1.14 & $(0.86-1.5)$ & 0.35 \\
\hline Mean Stanford Sleepiness Scale (SSS) & 1.70 & $(0.62-4.7)$ & 0.31 & 1.59 & $(0.36-7.0)$ & 0.54 \\
\hline Beck Depression Inventory score & 1.12 & $(0.97-1.3)$ & 0.13 & 1.11 & $(0.92-1.4)$ & 0.27 \\
\hline
\end{tabular}

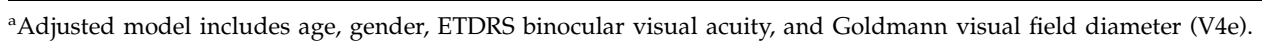

bStatistically significant $(P<0.05)$.

Table 1 compares the characteristics of subjects who noted photopsias frequently ( $>20 \%$ ) during test sessions to those who experienced them less frequently. The vast majority of males (89\%) had an increased frequency of photopsias, which was statistically significantly higher $(P=0.005)$ than that of females $(44 \%)$. We explored whether there were any statistically significant differences among males vs females for lab-based ETDRS VA $(P=0.96)$, Pelli-Robson CS $(P=0.69)$, GVF $(P=0.31)$, mean PC-based VA $(P=0.84)$, mean PC-based CS $(P=0.34)$, mean PC-based VF $(P=0.26)$, or the duration of vision loss $(P=0.59)$ or night vision loss $(P=0.40)$. We did not find evidence of any statistically significant differences in the level of vision according to gender, indicating that males and females were comparable according to their severity of RP. However, the males in this study on average reported statistically significantly greater levels of depressive symptoms on the BDI (7.4 points higher than females; 95\% CI: $1.8,13.1 ; P=0.01$ ). There was also a trend toward a significant difference according to gender for the mean PSS score (males 2.7 points higher than females; $95 \% \mathrm{CI}:-0.5,5.9 ; P=0.10$ ) and mean positive mood score (males 4.7 points lower than females; 95\% CI: $-9.8,0.5 ; P=0.07$ ).

There were no statistically significant differences in the frequency of photopsias in relation to subjects' age, vision, and sleepiness measures (ESS and SSS). Subjects with photopsias that occurred more frequently had a mean PSS score that was 4.3 points higher on average than those with less frequent photopsias (noted $<20 \%$ of the time) (95\% CI: $-1.7,-6.9 ; P=0.002)$. Individuals with photopsias that occurred more frequently had a mean positive mood score that was 7.7 points lower on average than those with less frequent photopsias (95\% CI: 2.8, 12.5; $P=0.003)$. Participants with photopsias that occurred more frequently had a mean negative mood score that was 2.7 points greater on average than those with less frequent photopsias (95\% CI: $-0.2,-5.3$; $P=0.04)$. The depressive symptoms score was 5.8 points greater on average for subjects with more frequent photopsias as compared with those with less frequent photopsias (95\% CI: 0.7, 10.7; $P=0.03$ ). 
Table 2 shows the crude and adjusted odds of frequent photopsias ( $>20 \%$ of test sessions) for each mean psychosocial variable. After adjusting for age, gender, VA, and GVF, for every 1-point increase in the mean perceived stress score, there was a statistically significant $57 \%$ increase in the odds of noting photopsias frequently (95\% CI: $1.04,2.4 ; P=0.03$ ). For every 1-point increase in the mean positive mood score, there was a statistically significant $38 \%$ decrease in the odds of noting photopsias frequently (95\% CI: 0.39, 0.98; $P=0.04$ ). There was a non-statistically significant trend for negative mood, such that for every 1-point increase in the mean negative mood score, there was a $44 \%$ increase in the odds of noting photopsias frequently (95\% CI: 0.95, 2.2; $P=0.09$ ). The odds of noting photopsias frequently did not increase statistically significantly according to the sleepiness measures or depressive symptoms.

Figure 1 provides a graphical depiction of the data, indicating a lower median and interquartile range for the mean positive mood score in subjects with photopsias during $>20 \%$ of sessions. Figure 1 also shows a higher median and interquartile range for the mean perceived stress score for those with photopsias noted during $>20 \%$ of sessions.

Table 3 displays the adjusted odds of experiencing photopsias at a test session in relation to 1 and 3 point changes in the psychosocial variables, as well as light exposure. There were no significant or qualitative differences in the crude vs adjusted odds in Table 3, and therefore only the adjusted odds are reported. After adjusting for age and gender, for every 3-point increase in perceived stress and positive mood score, there was a statistically significant $16 \%$ increase and $17 \%$ decrease in the odds of experiencing photopsias, respectively. The within-subject mean SD for the perceived stress and positive mood scores across test sessions was 4.5 points (range 1.3-10.8) and 5.1 points (range 1.6-8.7), respectively.

To further illustrate the scale of the psychosocial questionnaires, we determined relative changes from the mean for 1- and 3-point score changes. Across subjects, a 1-point score change was roughly equivalent to an $8 \%$ change from the mean for the perceived stress and positive mood scores, while 3-point score changes were roughly equivalent to 18 and $25 \%$ changes from the mean for the perceived stress and positive mood scores, respectively. Subjects had a change of at least 3 points from their mean score across $45 \%$ of sessions for the PSS and $49 \%$ of sessions for positive mood score. Figure 2 depicts the unadjusted increasing probabilities of photopsias with increasing perceived stress score and the decreasing probabilities of photopsias with increasing positive mood score.

The results in Table 3 are based on random intercept models. Although theoretically it may seem appropriate to allow each subject to have their own random slope in addition to a random intercept, as effects may vary across individuals, exploratory data analyses supported using a random intercept only model for the final analyses. The coefficients from the random slope and intercept models were not significantly or qualitatively different than the random intercept only models.

Negative mood, sleepiness, and light exposure (dim or bright) in the past hour were not statistically significantly associated with the occurrence of photopsias at a test session. Subjects had a change of at least 3 points from their mean score across $38 \%$ of sessions for negative mood score, $21 \%$ of sessions for the ESS, and $2 \%$ of sessions for the SSS. Across all subjects, 27 and $17 \%$ of the $\mathrm{PC}$ tests were taken after dim and bright light exposure in the past hour, respectively.
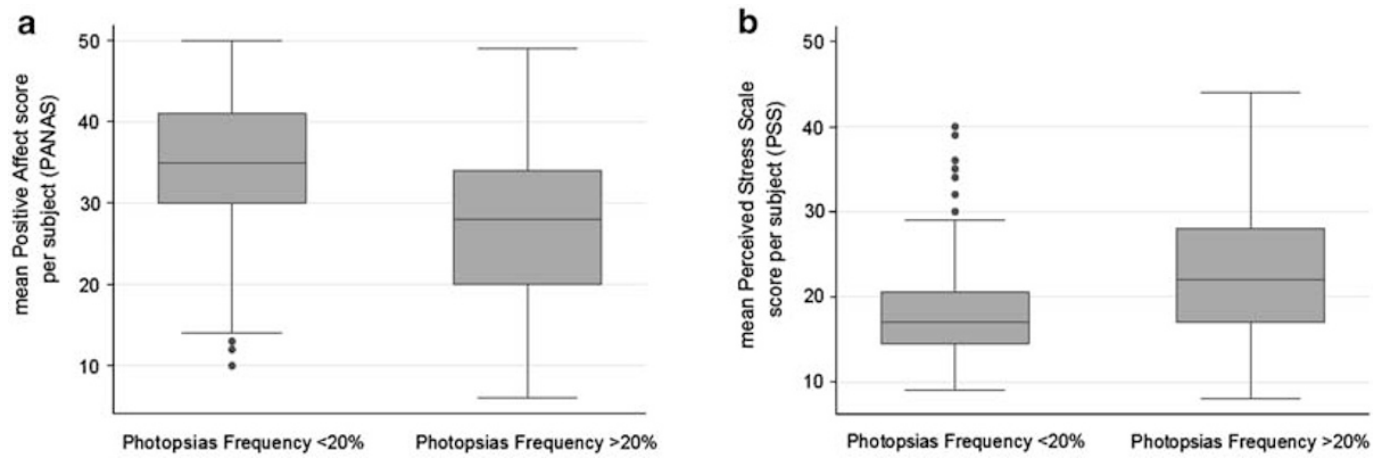

Figure 1 Box plots of the frequency of photopsias in relation to (a) positive mood and (b) perceived stress. The bottom and top of the box are the 25th and 75th percentiles (the lower and upper quartiles, respectively), and the band near the middle of the box is the 50th percentile (the median). The ends of the whiskers represent the the lowest datum within 1.5 times the interquartile range of the lower quartile, and the highest datum still within 1.5 times the interquartile range of the upper quartile. Any data not included between the whiskers are plotted as an outlier indicated by a dot. 
Table 3 Adjusted $^{\text {a }}$ odds of RP subjects $(n=36)$ experiencing photopsias at a test session

\begin{tabular}{lccccccc}
\hline & \multicolumn{3}{c}{ 1-point score increase } & & \multicolumn{3}{c}{ 3-point score increase } \\
\cline { 2 - 4 } \cline { 5 - 6 } & OR & $95 \%$ CI & P-value & & OR & $95 \%$ CI & P-value \\
\hline Perceived Stress Scale (PSS) & 1.05 & $(1.004-1.10)$ & $0.048^{\mathrm{b}}$ & & 1.16 & $(1.01-1.33)$ & $0.048^{\mathrm{b}}$ \\
Positive Mood (PANAS) & 0.94 & $(0.90-0.98)$ & $0.004^{\mathrm{b}}$ & & 0.83 & $(0.73-0.94)$ & $0.004^{\mathrm{b}}$ \\
Negative Mood (PANAS) & 1.00 & $(0.95-1.06)$ & 0.99 & & 1.00 & $(0.86-1.19)$ & 0.99 \\
Epworth Sleepiness Scale (ESS) & 1.01 & $(0.95-1.08)$ & 0.72 & & 1.04 & $(0.86-1.26)$ & 0.72 \\
Stanford Sleepiness Scale (SSS) & 1.00 & $(0.80-1.26)$ & 0.98 & & 1.01 & $(0.51-2.0)$ & 0.98 \\
Dim light exposure (vs medium or bright) & 0.73 & $(0.41-1.28)$ & 0.27 & & & \\
Bright light exposure (vs medium or dim) & 0.76 & $(0.37-1.58)$ & 0.47 & & & \\
\hline
\end{tabular}

adjusted model includes age and gender.

bstatistically significant $(P<0.05)$.
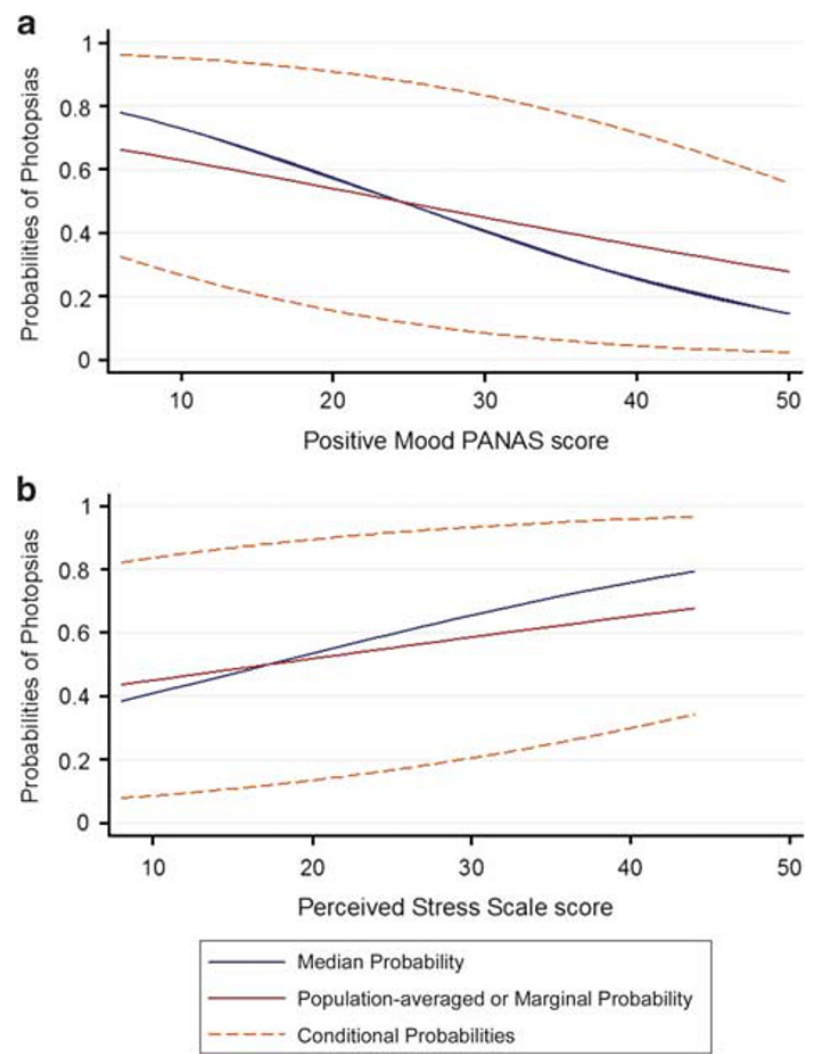

Figure 2 Probability of experiencing photopsias in relation to (a) positive mood and (b) perceived stress.

We were able to determine whether within-session variability occurred during VA and CS as these tests measured two thresholds simultaneously and independently through Bayesian estimation within a single session. We examined whether the presence of photopsias affects test performance within session. During CS testing, at times when there was no withinsession variability (ie, the two thresholds were the same; during $31 \%$ of all sessions), subjects were less likely to report photopsias ( $12 \%$ of all sessions) $\left(\chi^{2}\right.$ test: $\left.P=0.01\right)$.
At times when there was no within-session test variability, on average the mean CS was better by 0.023 $\log$ CS (95\% CI: $-0.04,-0.003 ; P=0.02)$. However, at times when the subjects reported photopsias during the vision tests, we did not find a statistically significant reduction in CS on average. We also did not find any statistically significant effect on VF, VA, or within-session variability of VA when photopsias were noted.

\section{Discussion}

In the present study, we have shown that both an increased frequency of photopsias across sessions and the occurrence of photopsias at each PC-based vision test session were statistically significantly associated with increased perceived stress and decreased positive mood. Higher positive mood reflects a state of greater energy, increased concentration, and pleasurable engagement, whereas lower positive mood is characterized by sadness and lethargy. Positive mood tends to be related to social activity and satisfaction, as well as to the frequency of pleasant events. ${ }^{11}$ We did not find any significant relationships with sleepiness, light exposure in the past hour, or subjects' age or level of vision.

Some may consider it an unexpected finding that positive but not negative mood was related to occurrence of photopsias. However, this finding is less surprising given that it has been previously reported that positive and negative mood measured with the PANAS are very weakly, negatively correlated, and are relatively independent both within- and between-subjects. ${ }^{15}$ Another previous study found that the dispositional components of positive and negative mood were independent, while the occasion- or situation-specific aspects of positive and negative mood were significantly correlated. ${ }^{16}$ Therefore, perhaps the difference in our findings for positive and negative mood reflects trait or dispositional aspects of mood reported by the subjects, rather than occasion-specific components. 
We were not able to confirm the previously surveyed $\mathrm{RP}$ patient reports of an association between photopsias and fatigue. Sleepiness scales, such as the SSS and ESS used in the current study, depend entirely on the individual's perception of the problem. It has been previously proposed that the construct measured by the ESS is uncertain, and it may be something other than daytime sleepiness or sleep propensity. ${ }^{17,18}$ Another limitation of the ESS scale is that it asks subjects to imagine themselves in situations they may actually rarely or never experience. Lastly, a recent paper determined that fatigue and sleepiness are two distinct constructs. ${ }^{19}$ Sleepiness refers to the propensity for spontaneous sleep onset, while fatigue indicates that it is difficult to maintain motor or mental energy levels, and may recover with rest, not necessarily sleep. We therefore would recommend future studies to evaluate the relationship between photopsias and fatigue measured by the Fatigue Severity Scales ${ }^{20}$ rather than sleepiness.

The current study did not find that the amount of light exposure in the past hour as reported by the subjects was related to the occurrence of photopsias during the PC- tests. We hypothesized that dim or bright light exposure may increase photopsias, on the basis of previous RP subjects' survey responses. Perhaps, changes in lighting have a more immediate effect on photopsias, and the amount of time that we inquired about was too long. Alternatively, a quantitative measure of light exposure using a lux meter may be a more robust measure than the participants' subjective ratings of bright, medium, and dim.

The retinal $v s$ cortical origin of photopsias in retinal degeneration patients has been a topic of discussion for over a century. ${ }^{5,21}$ In RP, it has been hypothesized that photopsias may be manifestations of spontaneous activity in degenerating retinal cells due to remodeling in the inner retina. ${ }^{22}$ There is an increased likelihood that intact neurons may discharge spontaneously due to lack of afferent impulses when there is disruption of the normal interaction between generation of an action potential, discharge, and inhibition. ${ }^{5}$ It has also been hypothesized that photopsias may be the release phenomena stemming from lack of inhibitory visual input. It is an interesting finding that psychological factors are related to photopsias; however, we were unable to determine in the present study design whether physiological factors related to changes in stress or positive mood contribute to these phenomena.

Over two-thirds of previously surveyed RP patients indicated that photopsias interfered with their vision. ${ }^{3}$ A limitation of the present study is that we did not ask the subjects to indicate during which vision test(s) they experienced the photopsias, and whether the photopsias were in a location that would have interfered with the test. This limited our ability to demonstrate whether vision test performance was impaired by the presence of certain types of photopsias. Photopsias can occur in a vast variety of situations and can take on several different forms and durations across RP patients, thereby making it difficult to determine their impact on patients' visual function.

During VA, CS, and VF assessments, some RP patients will report that photopsias interfere with their test performance, which is an important consideration when attempting to obtain consistent measures during clinical trials. RP patients implanted with retinal prostheses also note these phenomena and may have difficulty discriminating them from electrical stimulation provided by the prosthesis. Therefore, future research should attempt to measure and reduce the interference of photopsias in these situations. In addition, future research should focus on mechanisms involved in these phenomena, and determine whether increased stress and photopsias are related to RP disease progression.

Prospective designs, such as the one reported here, are important when studying this type of visual phenomena because retrospective studies may underestimate photopsias, if patients fail to notice, deny, or forget them. Many RP patients are surprised to learn that their photopsias are related to their RP and that others with RP also experience photopsias. The present study helps patients and providers understand some factors that contribute to an increased tendency to experience photopsias in RP. This study also suggests the need to test whether interventions involving mind-body therapies for the reduction of negative psychosocial states may reduce the frequency of photopsias in RP.

\section{Summary}

\section{What was known before}

- In a previous survey, retinitis pigmentosa patients indicated that the top three factors that tend to increase photopsias were bright light, fatigue, and stress; however, these factors have not been systematically studied in relation to photopsias in RP.

What this study adds

- In the present study, we have shown that both an increased frequency of photopsias across sessions and the occurrence of photopsias at each PC-based vision test session were statistically significantly associated with decreased positive effect and increased perceived stress. We did not find any significant relationships with sleepiness, light exposure in the past hour, or subjects' age or level of vision.

\section{Conflict of interest}

The authors declare no conflict of interest. 


\section{Acknowledgements}

We wish to thank the Advanced Methods in the Design and Analysis of Cohort Studies instructors, Drs Chris Cox and Alvaro Munoz, at the Johns Hopkins School of Public Health, as well as the 2010 class members for their insightful and valuable contributions. The funding support was provided by NIH K23EY018356 to AKB; Johns Hopkins Center for Mind-Body Research (NIH R24AT004641). The sponsors or funding organizations had no role in the design, conduct, or analysis of this research.

\section{References}

1 Murtha T, Stasheff SF. Visual dysfunction in retinal and optic nerve disease. Neurol Clin 2003; 21(2): 445-481.

2 Heckenlively JR, Yoser SL, Friedman LH, Oversier JJ. Clinical findings and common symptoms in retinitis pigmentosa. Am J Ophthalmol 1988; 105: 504-511.

3 Bittner AK, Diener-West M, Dagnelie G. A survey of photopsias in self-reported retinitis pigmentosa: location of photopsias is related to disease severity. Retina 2009; 29(10): 1513-1521.

4 Lepore FE. Spontaneous visual phenomena with visual loss: 104 patients with lesions of retinal and neural afferent pathways. Neurology 1990; 40(3 Pt 1): 444-447.

5 Kölmel HW. Visual illusions and hallucinations. Baillieres Clin Neurol 1993; 2(2): 243-264.

6 Bahrami H, Melia M, Dagnelie G. Lutein supplementation in retinitis pigmentosa: pc-based vision assessment in a randomized double-masked placebo-controlled clinical trial. BMC Ophthalmol 2006; 6(1): 23.

7 Hoddes E, Zarcone V, Smythe H, Phillips R, Dement WC. Quantification of sleepiness: a new approach. Psychophysiology 1973; 10(4): 431-436.

8 Hoddes E, Dement WC, Zarcone V. The development and use of the stanford sleepiness scale. Psychophysiology 1972; 9: 150 .
9 Johns MW. A new method for measuring daytime sleepiness: the Epworth sleepiness scale. Sleep 1991; 14(6): 540-545.

10 Cohen S, Kamarck T, Mermelstein R. A global measure of perceived stress. J Health Soc Behav 1983; 24(4): 385-396.

11 Watson D, Clark LA, Tellegen A. Development and validation of brief measures of positive and negative affect: the PANAS scales. J Pers Soc Psychol 1988; 54(6): 1063-1070.

12 Spacapan S, Oskamp S. The Social Psychology of Health. Sage: Newbury Park, CA, 1988.

13 Molloy GN, Pallant JF, Kantas A. A psychometric comparison of the positive and negative affect schedule across age and sex. Psychol Rep 2001; 88(3 Pt 1): 861-862.

14 Beck AT, Ward CH, Mendelson M, Mock J, Erbaugh J. An inventory for measuring depression. Arch Gen Psychiatry 1961; 4: 561-571.

15 Watson D, Clark LA. Measurement and mismeasurement of mood: recurrent and emergent issues. J Pers Assess 1997; 68(2): 267-296.

16 Schmukle SC, Egloff B, Burns LR. The relationship between positive and negative affect in the positive and negative affect schedule. J Res Personality 2002; 36: 463-475.

17 Miletin MS, Hanly PJ. Measurement properties of the Epworth sleepiness scale. Sleep Med 2003; 4(3): 195-199.

18 Chervin RD. Epworth sleepiness scale? Sleep Med 2003; 4(3): 175-176.

19 Neu D, Mairesse O, Hoffmann G, Valsamis JB, Verbanck P, Linkowski $\mathrm{P}$ et al. Do 'sleepy' and 'tired' go together? Rasch analysis of the relationships between sleepiness, fatigue and nonrestorative sleep complaints in a nonclinical population sample. Neuroepidemiology 2010; 35(1): 1-11.

20 Krupp LB, LaRocca NG, Muir-Nash J, Steinberg AD. The fatigue severity scale: application to patients with multiple sclerosis and systemic lupus erythematosus. Arch Neurol 1989; 46(10): 1121-1123.

21 Weinberger LM, Grant FC. Visual hallucinations and their neuro-optical correlates. Arch Ophthal 1940; 23(1): 166-199.

22 Marc RE, Jones BW, Anderson JR, Kinard K, Marshak DW, Wilson JH et al. Neural reprogramming in retinal degeneration. Invest Ophthalmol Vis Sci 2007; 48(7): 3364-3371. 